\title{
PROPOSIÇÃO DE UM REATOR FOTOCATALÍTICO PARA DESTRUIÇÃO DE MICRORGANISMOS EM AMBIENTES INTERIORES
}

\author{
Márcia Matiko Kondo*, José Fábio F. Orlanda, Maria da Glória A. B. Ferreira e Marco Tadeu Grassi ${ }^{\#}$ \\ Departamento de Tecnologia Química, Universidade Federal do Maranhão, Campus Universitário do Bacanga, 65080-040 \\ São Luis - MA
}

Recebido em 13/2/02; aceito em 3/6/02

\begin{abstract}
PROPOSITION OF A PHOTOCATALYTIC REACTOR TO INACTIVATE AIRBORNE MICROORGANISMS. The TiO/UV photocatalytic reactor was investigated as an alternative system to inactivate airborne microorganisms. The experiments performed in the absence of the catalyst showed that direct photolysis was not efficient to destruct microorganisms, with only $30 \%$ of inactivation. Similar inactivation percentage was obtained using $\mathrm{TiO}_{2}$ in absence of UV radiation. The destruction of microorganisms present in a contaminated indoor atmosphere, using the combination of $\mathrm{TiO}_{2} / \mathrm{UV}$ was very efficient, reaching more than $98 \%$ of destruction.
\end{abstract}

Keywords: microorganism inactivation; photocatalytic reactor; $\mathrm{TiO}_{2} / \mathrm{UV}$ irradiation.

\section{INTRODUÇÃo}

Antes da década de 70, os problemas de saúde relacionados com a poluição do ar de ambientes interiores chamavam pouca atenção da comunidade científica. $\mathrm{O}$ interesse por este assunto tem crescido nos últimos anos, uma vez que as pessoas têm passado a maior parte do seu tempo em ambientes fechados ${ }^{1}$. As causas da poluição do ar de ambientes interiores podem ser decorrentes da má ventilação, da presença de material particulado, da contaminação química, especialmente por compostos orgânicos voláteis e, também, devido à contaminação microbiológica. A melhora na ventilação e o emprego de filtros apropriados podem amenizar tais problemas. No entanto, a presença de compostos orgânicos voláteis e microrganismos (bactérias, fungos e vírus), ainda que em pequenas concentrações, causa grande preocupação ${ }^{2,3}$.

Um grande número de sintomas está relacionado com a má qualidade do ar. O conjunto destes sintomas foi definido como Síndrome do Edifício Doente ${ }^{3,4}$. Os principais incluem dor de cabeça, náusea, irritação nos olhos, problemas no sistema respiratório, sonolência e fadiga ${ }^{5}$.

Inúmeros métodos têm sido propostos com o objetivo de mitigar o problema da contaminação biológica do ar interno, incluindo filtros para a remoção de materiais biológicos. Os filtros têm sido utilizados devido ao baixo custo e fácil manuseio, apresentando, entretanto, a desvantagem de não destruírem os microrganismos, e sim transferi-los para outro meio ${ }^{4}$.

Um processo alternativo para inativar os microrganismos, proposto no presente trabalho, emprega o sistema $\mathrm{TiO}_{2} / \mathrm{UV}$. No início da década de 70, Fujishima e $\mathrm{Honda}^{6}$ descobriram que a água era decomposta a hidrogênio e oxigênio na presença de um eletrodo de dióxido de titânio e luz UV. Quando o $\mathrm{TiO}_{2}$ é irradiado com luz UV com energia igual ou superior ao seu "bandgap" o par elétron/lacuna

\footnotetext{
* endereço atual: Departamento de Física e Química, Universidade Federal de Itajubá, Av. BPS, 1303, 37500-903 Itajubá - MG e-mail:mmkondo@unifei.edu.br

\# endereço atual: Departamento de Química, Universidade Federal do Paraná, CP 19081, 81531-990 Curitiba - PR
}

positiva é gerado na superfície da partícula deste semicondutor. Como consequiência, a lacuna positiva pode oxidar água adsorvida na superfície do $\mathrm{TiO}_{2}$, formando radicais hidroxila (HO'). Estes radicais são altamente reativos ${ }^{7-9}$, possuindo um elevado potencial de oxidação. Dentre as possibilidades de aplicação deste sistema, o emprego na área ambiental tem merecido maior destaque devido, principalmente, à possibilidade de alcançar a mineralização completa de vários compostos orgânicos potencialmente tóxicos ${ }^{10}$.

Embora a grande maioria dos estudos utilize o sistema $\mathrm{TiO}_{2} / \mathrm{UV}$ para a degradação de compostos orgânicos em soluções aquosas ${ }^{11-12}$, recentemente alguns pesquisadores têm dedicado esforços na utilização deste processo para destruir microrganismos ${ }^{13-15}$.

Ireland e colaboradores ${ }^{13}$ inativaram Escherichia coli em uma solução aquosa, na presença do fotocatalisador $\mathrm{TiO}_{2}$. Os autores verificaram que os radicais $\mathrm{HO}^{*}$ gerados por este sistema agiam como germicidas, devido ao seu alto potencial de oxidação e baixa seletividade. Um outro estudo mostrou que este sistema também é capaz de desativar esporos de B. pumilus presentes em fase aquosa ${ }^{16}$.

O presente trabalho visa avaliar a eficiência de um sistema fotocatalítico na destruição de microrganismos presentes em ambientes interiores contaminados. O sistema baseia-se no emprego do catalisador $\mathrm{TiO}_{2}$ em presença de irradiação UV.

\section{PARTE EXPERIMENTAL}

\section{O fotorreator}

$\mathrm{O} \mathrm{TiO}_{2}$ (anatase P-25, Degussa) foi imobilizado na superfície interna do fotorreator cilíndrico de vidro, com dimensões de 85,5 $\mathrm{cm}$ de comprimento e $3,5 \mathrm{~cm}$ de diâmetro interno. O processo de imobilização consiste no preparo de uma suspensão contendo $12 \mathrm{~g}$ de $\mathrm{TiO}_{2}, 4 \mathrm{~mL}$ de água destilada e $0,4 \mathrm{~mL}$ de acetilacetona (Aldrich) ${ }^{14}$. Esta suspensão foi homogeneizada por $10 \mathrm{~min}$ em banho de ultrasom e, em seguida, adicionou-se mais $25 \mathrm{~mL}$ de água e $0,2 \mathrm{~mL}$ de Triton ${ }^{\circledast}$ X-100. A suspensão resultante foi então aplicada ao reator e este foi seco à temperatura ambiente. Este processo foi repetido por mais três vezes. Uma lâmpada de luz negra fluorescente de $30 \mathrm{~W}$ de potência, com máximo de emissão em $365 \mathrm{~nm}$, foi introduzida no centro do reator. 
O fotorreator anular foi conectado à saída de um aparelho de ar condicionado de 7500 BTUs, conforme mostra o esquema da Figura 1. Todo o ar que saía do aparelho de ar condicionado era então forçado a passar pelo fotorreator.

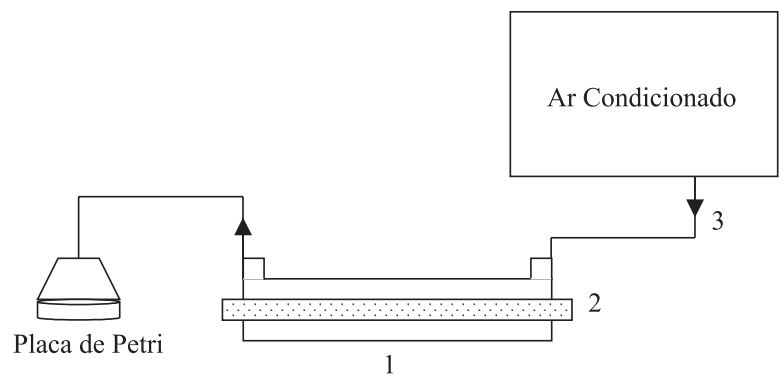

Figura 1. Ilustração esquemática do fotorreator contendo $\mathrm{TiO}_{2}$ imobilizado (1) e irradiado com luz UV (2). O ar da saída do aparelho de ar condicionado (3) é forçado a passar pelo reator e em seguida pela placa de Petri contendo meios de cultura (APC ou Agar Batata)

\section{Caracterização dos microrganismos}

Os microrganismos presentes (bactérias e fungos) foram caracterizados através do contato do ar contaminado com placas de Petri contendo os meios de cultura APC e Agar Batata durante 30 min. Após a exposição, as mesmas foram incubadas durante $48 \mathrm{~h}$ a uma temperatura de $37^{\circ} \mathrm{C}$. Todos os experimentos foram realizados em triplicatas.

Transcorrido o tempo necessário de incubação, as colônias formadas foram quantificadas. Para determinação taxonômica das bactérias presentes nas placas foram realizados testes microbiológicos baseados no método de $\mathrm{Gram}^{17}$.

\section{RESULTADOS E DISCUSSÃO}

Inicialmente foi realizado um acompanhamento para se verificar o tempo de crescimento dos microrganismos, conforme mostrado na Figura 2. Para tanto, o aparelho de ar condicionado foi mantido em funcionamento, sob uma vazão de $1200 \mathrm{~mL} \mathrm{~min}^{-1} \mathrm{e}$ o ar que passava por ele era coletado por até $45 \mathrm{~min}$ consecutivos. Através da Figura 2 é possível observar que a partir de 30 min não existe diferença acentuada no número de bactérias e fungos coletados. Em função

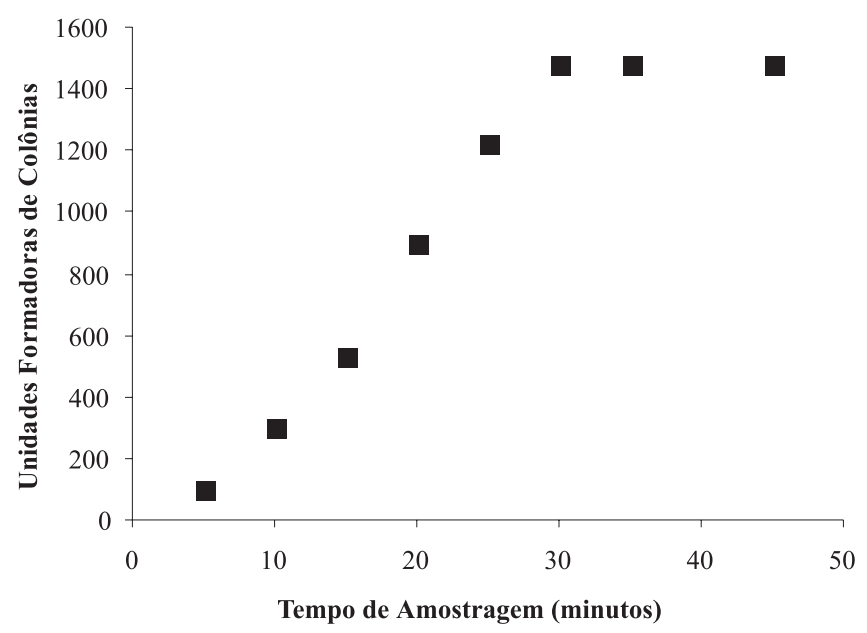

Figura 2. Quantidade de unidades formadoras de colônias coletadas, média de 3 experimentos, em função do tempo de exposição destes resultados, os experimentos subseqüentes foram realizados com as amostras de ar sendo coletadas por períodos de $30 \mathrm{~min}$.

A Figura 3 mostra as quantidades de unidades formadoras de colônias coletadas em quatro experimentos separados: somente radiação UV, somente $\mathrm{TiO}_{2}$, com a combinação de $\mathrm{TiO}_{2} / \mathrm{UV}$ e o controle, ou seja, na ausência de luz UV e do catalisador. Os microrganismos são apenas parcialmente destruídos pela fotólise direta. Os percentuais de inativação foram de $45 \%$ para bactérias e $30 \%$ para fungos. Em presença apenas do catalisador, $46 \%$ e $26 \%$ de bactérias e fungos foram inativados, respectivamente. Na realidade, estes percentuais de remoção são consequiência de um processo de adsorção dos microrganismos no $\mathrm{TiO}_{2}$ imobilizado na superfície do vidro. Esta adsorção, ou seja, o contato do catalisador com o microrganismo é necessário para que os microrganismos possam ser destruídos pelo processo fotocatalítico, uma vez que os radicais hidroxila se encontram na superfície do $\mathrm{TiO}_{2}{ }^{10,11}$. Os microrganismos adsorvidos na superfície do catalisador são totalmente destruídos quando irradiados com luz UV ${ }^{11}$. Este fato torna a superfície auto limpante, tornando-se desnecessária a manutenção constante do sistema.

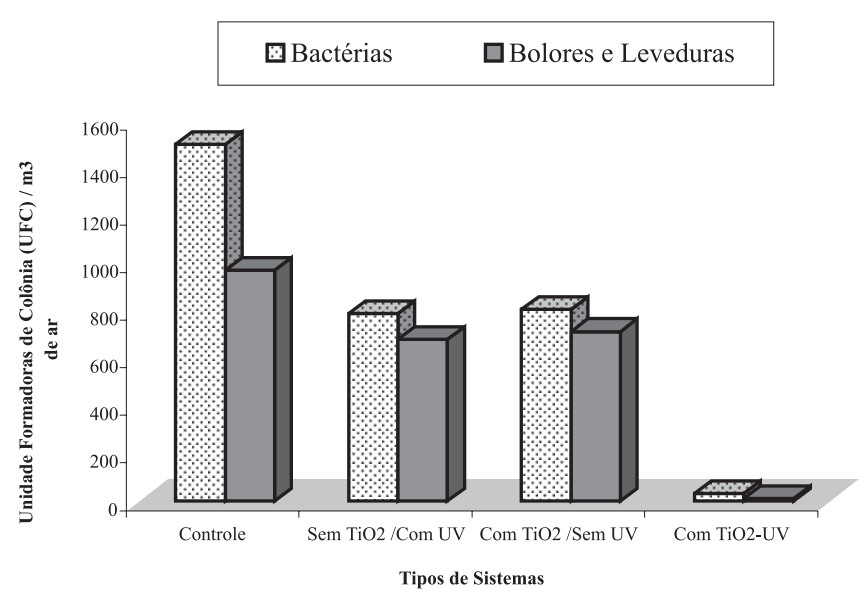

Figura 3. Quantidades de bactérias e fungos destruídos por: fotólise direta, em presença somente de $\mathrm{TiO}_{2}$, empregando o processo fotocatalítico $\mathrm{TiO}_{2} /$ UV, em comparação ao controle. Média de 3 experimentos

Quando o ar contaminado foi forçado a passar pelo reator fotocatalítico, verificou-se a completa inativação dos microrganismos. Os níveis de destruição, nestas condições, foram de $98 \%$ para bactérias e $99 \%$ para fungos.

Os estudos realizados não permitiram que os microrganismos pudessem ser identificados. Entretanto, uma avaliação taxonômica evidenciou que a maioria daqueles encontrados no ambiente de estudo eram Gram positivos.

\section{CONCLUSÕES}

Os resultados apresentados neste trabalho demonstram a possibilidade de se utilizar o presente sistema $\mathrm{TiO}_{2} / \mathrm{UV}$ na destruição de microrganismos presentes no ar de ambientes fechados. Sendo o $\mathrm{TiO}_{2}$ um catalisador, o reator requer manutenção mínima; no entanto, ainda são necessários estudos mais aprofundados para viabilizar o seu uso em escala comercial.

\section{AGRADECIMENTOS}

Os autores agradecem à FAPEMA pelo apoio financeiro. 


\section{REFERÊNCIAS}

1. Jones, A. P.; Atmospheric Environ. 1999, 33, 4535.

2. Neto, F. K.; Siqueira, L. F. G.; Brasindoor 1999, $2,1$.

3. Brickus, L. S. R.; Aquino Neto, F. R.; Quim. Nova 1999, 22, 65

4. Goswani, D. Y; Triverdi, D. M.; Block, S. S.; Trans. of the ASME, 1997, $119,92$.

5. Kostiainen, R.; Atmospheric Environ. 1995, 29, 693.

6. Fujishima, A.; Honda, K.; Nature 1972, 238, 37.

7. Matthews, R. W.; Wat. Res. 1986, 20, 569.

8. Pruden, A. L.; Ollis, D. F.; J. Catal. 1983, 82, 404.

9. Smart, L.; Moore, E.; Solid State Chemistry - An Introduction, Chapman \& Hall: New York, 1992.

10. Jardim, W. F.; Moraes, S. G.; Takiyama, M. M. K.; Wat. Res. 1997, 31, 1728.
11. Fujishima, A.; Rao, T. N.; Tryk, D. A.; J. Photochem. Photobiol. 2000, 1,

12. Takiyama, M. M. K.; Tese de Doutourado, Department of Civil and Environmental Engineering, University of Delaware, USA, 1996.

13. Ireland, J. C.; Klostermann, P.; Rice, E. W.; Clark, R. M.; Appl. Environ. Microbiol. 1993, 59, 1668.

14. Jacoby, W. A.; Maness, P. C.; Wolfrum, E. J.; Blake, D. M.; Fennell, J.A.; Environ. Sci. Technol. 1998, 32, 2650.

15. Saito, T; Iwase. T.; Horie, J.; Morioka, T.; J. Photochem. Photobiol. B. Biol. 1992, 81,37

16. Pham, H. N.; McDowell, T.; Wilkins, E.; J. Environ. Sci. Health 1995, A30, 627.

17. Pelczar Jr., M. J.; Chan, E. C. S.; Krieg, N. R.; Microbiology Concepts and Applications, McGraw-Hill: New York, 1993. 